\title{
Yield of Muscle Biopsy in Patients with Findings of Myopathy on Electrodiagnostic Testing
}

\author{
Sarah Hasan Siddiqui ${ }^{1}$ Raheel Ahmed ${ }^{1}$ Safia A
${ }^{1}$ Department of Neurophysiology, Aga Khan University, Karachi, \\ Pakistan \\ 2Department of Medicine, Aga Khan University, Karachi, Pakistan
}

\author{
Ambreen Zain ${ }^{1}$ Sara Khan ${ }^{1}$
}

J Neurosci Rural Pract 2019;10:489-493

\begin{abstract}
Background The evaluation of neuromuscular diseases includes detailed clinical assessment, blood testing, electrodiagnostic studies (EDS), biopsy, and genetic tests. EDS alone cannot provide a specific diagnosis. Further testing in the form of genetic tests or muscle biopsy (MB) is required.

Objective The objective of the study is to evaluate the yield of MB in patients with findings of myopathy on electrodiagnostic testing and assess the factors affecting an abnormal biopsy outcome.

Methods Electromyography (EMG)/nerve conduction studies (NCS) performed for suspected myopathy over 5 years from 2011 to 2016, at the neurophysiology department of a tertiary care center in Pakistan, were reviewed. Based on inclusion criteria, records of 58 patients were retrospectively reviewed.

Results After an EMG/NCS diagnosis of myopathy, the frequency of MB testing was only $10.1 \%$. The median age of patients was 26.5 years. The clinically suspected diagnosis was categorized into hereditary myopathy $(n=15,25.9 \%)$ and acquired myopathy ( $n=18,31 \%)$. The positive predictive value of EMG is $77.2 \%$. Twenty-eight ( $48.2 \%$ ) patients had abnormal MB whereas 20 (34.4\%) revealed normal findings. Factors significantly influencing an abnormal outcome of biopsy included moderate-to-severe

Keywords

- creatine kinase

- electromyography

- nerve conduction study

- muscle biopsy

- myopathy elevation of creatine kinase $(>2,000 \mathrm{U} / \mathrm{L})$, presence of denervation changes, and severe myopathy on EMG.

Conclusion Even though the overall yield of MB testing may not be very high in our setting due to the unavailability of special techniques and expertise, certain factors can help to improve the diagnostic yield. Clinicians should encourage MB testing, especially in cases with strong clinical, laboratory and electrodiagnostic suspicion, and absence of genetic testing for suspected myopathy.
\end{abstract}

\section{Introduction}

Accurate diagnosis of neuromuscular disorders (NMDs) is based on a detailed clinical assessment, laboratory and electrodiagnostic testing, histological examination of tissue, and genetic tests. ${ }^{1-3}$ Nerve conduction studies (NCS) and electromyography (EMG) are valuable in localizing and assessing the severity of NMDs, identifying the distribution of involved muscles, and the appropriate muscle to biopsy, and may be useful for assessing disease progression. While NCS/EMG can suggest a myopathic process, it is seldom useful to provide a specific diagnosis, may be technically difficult, especially in children and may be subject to operator bias. Therefore, further testing in the form of muscle biopsy (MB) or genetic testing is required. 4,5

MB testing is dependent on proper sampling technique/ handling, muscle selection, expertise of the pathologist, availability of numerous stains, and electron microscopic 
evaluation. ${ }^{6,7}$ In Pakistan, we face limitations in all of these steps. As a result, clinicians have a tendency to rely on clinical judgment and avoid an MB to spare patient time, pain, and money.

With these limitations, data on the diagnostic yield of $\mathrm{MB}$ in myopathic disorders are lacking from Pakistan. We planned this study to determine the yield of MB in clinically, and EMG diagnosed cases of myopathy.

\section{Methods}

After approval from Institutional Ethics Review Committee, 1,290 electrodiagnostic studies (EDS) performed for suspected myopathy over 5 years from 2011 to 2016, at the neurophysiology department of a large private tertiary care university hospital of Pakistan, were reviewed. Of these, 570 studies showed evidence of myopathy which was based on short-mean duration and short amplitude of motor units in two or more muscles. Only 58 of these underwent MB testing in the same hospital and were included in the study. Medical records of these selected cases were retrospectively reviewed for demographic, clinical, laboratory, and histopathological data.

Clinical data included the suspected diagnosis which was categorized into hereditary or acquired myopathy, rate of progression of myopathy, and the predominant distribution of weakness.

Electrodiagnostic variables included the presence of spontaneous activity (positive sharp waves and fibrillation potentials) and the severity of myopathy on needle examination. The latter was categorized into mild-to-moderate and severe myopathy based on the electromyographer's assessment. Motor units of short duration and amplitude intermixed with normal looking motor units were categorized into mildmoderate category, whereas severe category included only myopathic units without any evidence of intermixed normal units.

MB data were classified into three main groups, negative for myopathy (normal), positive (inflammatory or dystrophic), and inconclusive.

Data were compiled and analyzed using IBM SPSS Statistics for Windows Version 19.0 (IBM Corp., Armonk, NY). The histopathological diagnosis of muscle biopsies was compared with the clinically suspected diagnosis, EMG, and serological findings using the Chi-square test.

\section{Results}

Of the 570 patients that had a positive EMG for myopathy during the 5 years' study, only 58 underwent MB testing $(10.1 \%)$. Patients of all age groups were enrolled in the study with a median age of 26.5 years (interquartile range $=14-42.2$ ).

Fifteen cases were clinically suspected of having a hereditary myopathy, while 18 were suspected to have an acquired myopathy, which included inflammatory myopathy in 17 and critical illness myopathy in one patient. Muscle enzymes, that is, creatine kinase $(\mathrm{CK})$ and aldolase levels were checked in 48 and 21 patients, respectively. About 37.5\% had moderate-to-severe elevation of CK (>2,000 U/L). On NCS/EMG, severe myopathy was noted in 29 (50\%) of cases, and denervation changes suggestive of a necrotizing myopathy were seen in 35 (60.3\%) patients. One patient showed myotonic discharges on EMG with mild-to-moderate severity of myopathy electrically. Histopathological analysis of MB turned out to be normal in this patient (-Table $\mathbf{1}$ ).

Out of 58 patients with myopathic findings on electrodiagnostic testing who had MB, 28 (48.2\%) had an abnormal biopsy result. Sixteen (57.14\%) of the abnormal muscle biopsies showed inflammatory pathology, 11 (39.2\%) had dystrophic biopsies of which 3 were dystrophic other than dystrophinopathy and 1 patient showed evidence of steroid-induced myopathy (-Fig. 1).

Certain findings on electrodiagnostic tests may have an influence on the outcome of MB (-Table 2). Out of the 22 patients with severe myopathy on EMG, 17 (77.3\%) revealed an abnormal MB, whereas of those with a mild to moderate myogenic needle examination, 15 (57.7\%) showed normal muscle histopathology. This difference was statistically significant $(p=0.02)$. In addition, the presence of fibrillation potentials and positive sharp waves correlated with a positive MB in $20(71.4 \%)$ cases ( $p=0.04)$. Similarly, the degree of elevation of CK correlated significantly with the biopsy result as moderate-to-severe elevation of CK was noted in 75\% (12) of abnormal biopsies ( $p=0.02)$.

Of the 15 patients suspected of a hereditary myopathy, 6 (40\%) had dystrophic muscle biopsies. On the other hand, 12 out of 18 patients (66.7\%) with an acquired myopathy yielded a biopsy outcome correlating with the clinical suspicion. A significant number of muscle biopsies revealed normal histopathology when the clinical diagnosis was not specified, that is, two out of four (50\%) or not available (11 out of $20,55 \%)$. Overall, the correlation of clinically suspected diagnosis with the biopsy result did show statistical significance ( $p=0.002$; - Table 3$)$.

The severity of myopathy on needle examination, the presence of denervation changes, and the severity of CK elevation, although predictive of an overall positive biopsy result, were not very useful in terms of predicting a specific histopathological diagnosis and were found in both dystrophic and inflammatory muscle biopsies ( $p=0.05,0.07$, and 0.14, respectively; - Table $\mathbf{3}$ ).

\section{Discussion}

Genetic testing and/or histopathological analysis of muscles are considered to be definitive tests for the diagnosis of myopathic disorders with clinical, serological, and electrodiagnostic data providing supporting evidence. Given the limited availability of genetic testing in Pakistan, much of the reliance is on clinical and electrodiagnostic evaluations. $\mathrm{MB}$ testing although available in certain tertiary care centers is poorly relied on by clinicians due to the lack of expertise and special techniques. This is evident from the finding that out of a total of 570 EMG studies which showed myopathic process, only $10.1 \%$ underwent MB. Even though we were not 
Table 1 Characteristics of the study population

\begin{tabular}{|c|c|}
\hline Characteristics & $n(\%)$ \\
\hline \multicolumn{2}{|l|}{ Age (y) } \\
\hline Mean & $30.6 \pm 1.96$ \\
\hline Median (IQR) & 26.5.(14-44.2) \\
\hline \multicolumn{2}{|l|}{ Gender } \\
\hline Male & $33(56.9)$ \\
\hline Female & $25(43.1)$ \\
\hline \multicolumn{2}{|l|}{ Rate of progression } \\
\hline Rapid & $5(8.6)$ \\
\hline Moderate & $21(36.2)$ \\
\hline Slow & $22(37.9)$ \\
\hline Unknown & $10(17.2)$ \\
\hline \multicolumn{2}{|l|}{ Distribution of weakness } \\
\hline Predominant proximal & $41(70.6)$ \\
\hline Predominant distal & $9(15.5)$ \\
\hline Not available & $8(13.8)$ \\
\hline EMG denervation & $35(60.3)$ \\
\hline \multicolumn{2}{|l|}{ EMG severity } \\
\hline Mild & $11(19)$ \\
\hline Moderate & $18(31)$ \\
\hline Severe & $29(50)$ \\
\hline \multicolumn{2}{|l|}{ EMG distribution } \\
\hline Generalized & $27(46.6)$ \\
\hline Proximal & $30(51.7)$ \\
\hline Distal & $1(1.7)$ \\
\hline \multicolumn{2}{|l|}{ Serum $\mathrm{CK}^{\mathrm{a}}$} \\
\hline Mean & $2,063.08 \pm 3,050.1$ \\
\hline Median (IQR) & $700.5 .(141-2,502.2)$ \\
\hline Normal $(<200)$ & $14(24.1)$ \\
\hline Mild $(201-2,000)$ & $16(27.6)$ \\
\hline $\begin{array}{l}\text { Moderate-to-severe } \\
(>2,000)\end{array}$ & $18(31.0)$ \\
\hline \multicolumn{2}{|l|}{ Serum aldolase ${ }^{\mathrm{b}}$} \\
\hline Mean & $19.9 \pm 22.9$ \\
\hline Median (IQR) & 10.(5.1-28.7) \\
\hline \multicolumn{2}{|l|}{$\mathrm{ESR}^{\mathrm{c}}$} \\
\hline Mean & $29.8 \pm 30.5$ \\
\hline Median (IQR) & $21 .(5-47)$ \\
\hline \multicolumn{2}{|l|}{ Clinical diagnosis } \\
\hline Hereditary myopathy & $15(25.9)$ \\
\hline Acquired myopathy & $18(31)$ \\
\hline Not specified & $5(8.6)$ \\
\hline Not available & $20(34.5)$ \\
\hline
\end{tabular}

(continued)
Table 1 (continued)

\begin{tabular}{|l|l|}
\hline Characteristics & $\boldsymbol{n}(\%)$ \\
\hline Biopsy site & \\
\hline Deltoid & $21(26.2)$ \\
\hline Biceps brachii & $18(31)$ \\
\hline Quadriceps femoris & $15(25.9)$ \\
\hline Gastrocnemius & $1(1.7)$ \\
\hline
\end{tabular}

Abbreviations: CK, creatine kinase; EMG, electromyography; ESR, erythrocyte sedimentation rate; $I Q R$, interquartile range.

aData available for 48 patients.

'Data available for 21 patients.

'Data available for 31 patients.

able to account for those patients who may have had an MB from a different center, the frequency of MB testing in our setting still appears to be very low. This study was performed to estimate the overall yield of MB, even with above-mentioned limitations, in our setup.

We found that out of a total of 58 muscle biopsies done during 2011 to 2016, 28 (48.27\%) provided a positive result of which $19 \%$ were dystrophinopathy, $27.6 \%$ were inflammatory, and one showed findings of steroid-induced myopathy. The positive diagnostic yield of MB testing reported in different studies ranges from 60 to $93 \%$., $, 5,8-10$

The relatively low rate of positive results on the histopathological analysis in our study can be because of numerous factors. Improper muscle selection for biopsy, inadequate amount of the biopsy specimen, lack of use of special stains, and electron microscopic examination, limited experience of the observer or very advanced stage at the time of biopsy testing may contribute to the biopsy outcomes. ${ }^{6,7}$

Ten cases (17.2\%) showed minimal findings for a definitive pathological diagnosis. This is almost similar to the finding noted by Rabie et al, where 5 out of the 55 patients showed nonspecific biopsy findings despite a myopathic EMG. ${ }^{4}$ Advanced disease stage, biopsy testing after treatment initiation, or inadequate sampling technique may contribute to these inconclusive results.

In $34.4 \%, \mathrm{MB}$ turned out to be normal despite the electrodiagnostic evidence of a myopathy. Of these, nine had a strong clinical suspicion of myopathy, five had evidence of a severe myopathy on EMG, eight had active denervation changes on EMG, and eight had elevated muscle enzyme, CK (four had $\mathrm{CK}>2,000 \mathrm{U} / \mathrm{L}$ ). These findings raise the possibility of a false-negative MB in these cases which can be because of the factors mentioned above.

In our study, the positive predictive value of EMG was found to be $77.2 \%$. The sensitivity of EMG for diagnosing myopathy ranges between 58 and 90\% in different studies. These figures may vary among different muscles being tested, at different disease stages and in different age groups. It, however, tends to be lower (14-75\%) in children with age $<2$ years. ${ }^{2,4,9,11}$ This wide variation of sensitivity of EMG among different studies probably suggests that evaluation of motor 


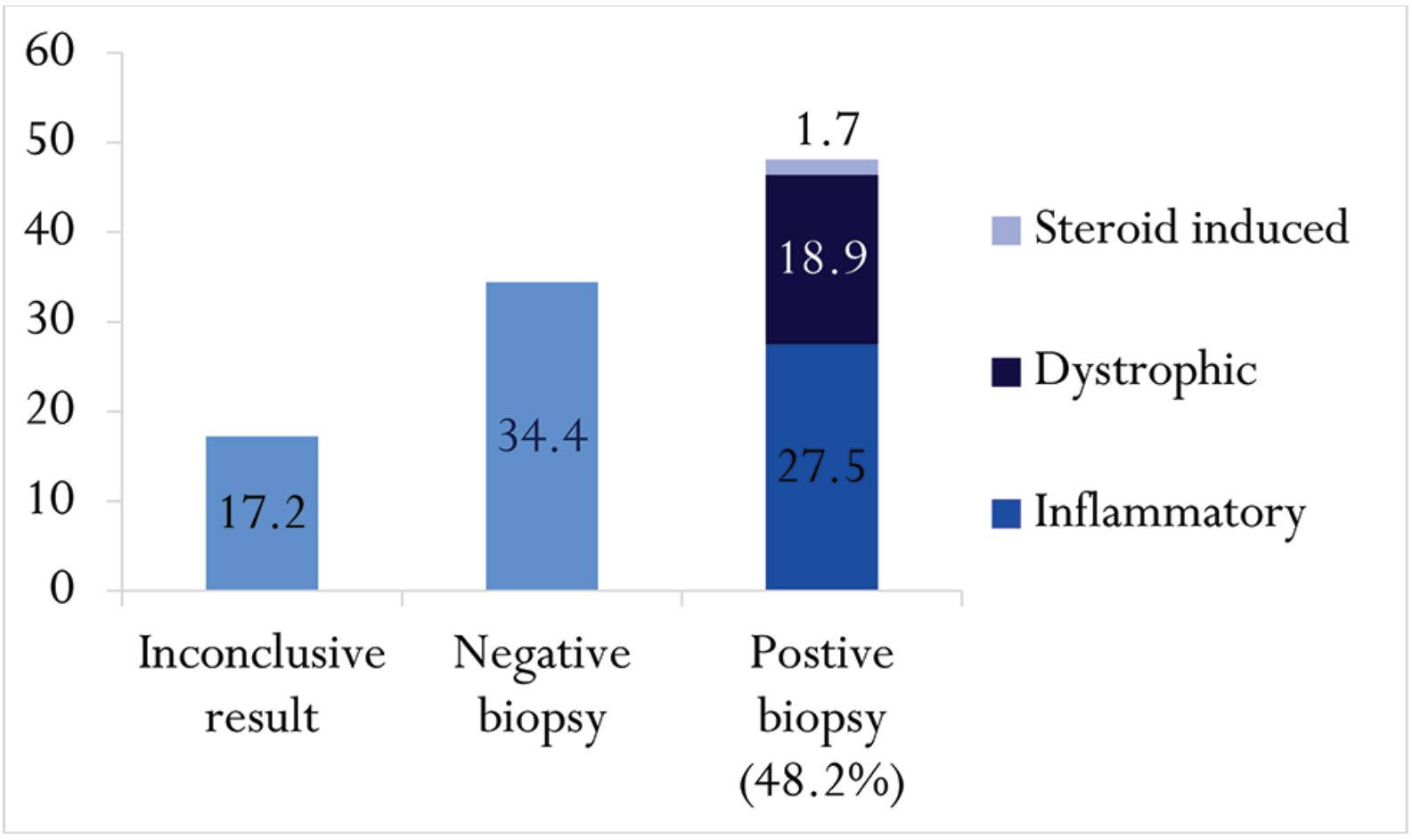

Fig. 1 Histopathological outcome of muscle biopsy (\%).

Table 2 Comparison of muscle biopsy findings with clinical, laboratory, and electrodiagnostic data ${ }^{a}$

\begin{tabular}{|c|c|c|c|}
\hline Data & $\begin{array}{l}\text { Positive biopsy }(n=28) \\
n(\%)\end{array}$ & $\begin{array}{l}\text { Negative biopsy }(n=20) \\
n(\%)\end{array}$ & $p$-Value \\
\hline \multicolumn{4}{|l|}{ EMG denervation } \\
\hline Yes & $20(71.4)$ & $8(28.6)$ & \multirow{2}{*}{0.04} \\
\hline No & $8(40)$ & $12(60)$ & \\
\hline \multicolumn{4}{|l|}{ Serum CK } \\
\hline Normal $<200$ & $3(25)$ & $9(75)$ & \multirow{3}{*}{0.02} \\
\hline Mild 201-2000 & $8(66.7)$ & $4(33.3)$ & \\
\hline Moderate-to-severe $>001$ & $12(75)$ & $4(25)$ & \\
\hline \multicolumn{4}{|l|}{ EMG severity } \\
\hline Mild to moderate & $11(42.3)$ & $15(57.7)$ & \multirow{2}{*}{0.02} \\
\hline Severe & $17(77.3)$ & $5(22.7)$ & \\
\hline \multicolumn{4}{|l|}{ Clinical diagnosis } \\
\hline Hereditary & $7(63.6)$ & $4(36.4)$ & \multirow{4}{*}{0.06} \\
\hline Acquired & $13(81.2)$ & $3(18.8)$ & \\
\hline Not specified & $2(50)$ & $2(50)$ & \\
\hline Not available & $6(35.3)$ & $11(64.7)$ & \\
\hline
\end{tabular}

Abbreviations: CK, creatine kinase; EMG, electromyography.

anconclusive biopsies were not included in this analysis.

units on needle electrode examination is a subjective assessment of the operator and is subject to interobserver variability with both false-positive and false-negative findings. This is, therefore, the reason that the diagnostic accuracy of EMG for myopathy is reported to be around 60\%. ${ }^{2}$ As EMG testing can have false-positive results, therefore, a negative $\mathrm{MB}$ in cases of mild or even moderate myopathy on EMG testing should be interpreted in accordance with other clinical and serological evidence.

\section{Conclusion}

Based on the findings from this study, it can be suggested that, even though the overall yield of MB testing may be 
Table 3 Comparison of biopsy findings with clinical, laboratory, and electrodiagnostic data ${ }^{a}$

\begin{tabular}{|c|c|c|c|c|c|}
\hline Data & $\begin{array}{l}\text { Inconclusive }(n=10) \\
n(\%)\end{array}$ & $\begin{array}{l}\text { Inflammatory }(n=16) \\
n(\%)\end{array}$ & $\begin{array}{l}\text { Dystrophic }(n=11) \\
n(\%)\end{array}$ & $\begin{array}{l}\text { Normal }(n=20) \\
n(\%)\end{array}$ & $p$-Value \\
\hline \multicolumn{6}{|l|}{ EMG severity } \\
\hline Mild to moderate & $3(10.3)$ & $6(20.7)$ & $5(17.2)$ & $15(51.7)$ & \multirow{2}{*}{0.05} \\
\hline Severe & $7(25)$ & $10(35.7)$ & $6(21.4)$ & $5(17.9)$ & \\
\hline \multicolumn{6}{|l|}{ EMG denervation } \\
\hline Yes & $7(20.6)$ & $13(38.2)$ & $6(17.6)$ & $8(23.5)$ & \multirow{2}{*}{0.07} \\
\hline No & $3(13)$ & $3(13)$ & $5(21.7)$ & $12(52.2)$ & \\
\hline \multicolumn{6}{|l|}{ Serum CK } \\
\hline Normal <200 & $2(14.3)$ & $2(14.3)$ & $1(7.1)$ & $9(64.3)$ & \multirow{3}{*}{0.14} \\
\hline Mild 201-2000 & $4(26.7)$ & $5(33.3)$ & $2(13.3)$ & $4(26.7)$ & \\
\hline $\begin{array}{l}\text { Moderate to } \\
\text { severe }>2001\end{array}$ & $2(11.1)$ & 7 (38.9) & $5(27.8)$ & $4(22.2)$ & \\
\hline \multicolumn{6}{|l|}{ Clinical diagnosis } \\
\hline Hereditary & $4(26.7)$ & $1(6.7)$ & $6(40)$ & $4(26.7)$ & \multirow{4}{*}{0.002} \\
\hline Acquired & $2(11.2)$ & $12(66.7)$ & $1(5.6)$ & $3(16.7)$ & \\
\hline Not specified & $1(25)$ & $1(25)$ & 0 & $2(50)$ & \\
\hline Not available & $3(15)$ & $2(10)$ & $4(20)$ & $11(55)$ & \\
\hline
\end{tabular}

Abbreviations: CK, creatine kinase; EMG, electromyography.

${ }^{a}$ One patient with steroid-induced myopathy was excluded from this analysis as it was a separate category.

lower as compared to other countries, certain factors can help to improve the yield. They include moderate-to-severe CK elevation, severe myopathy, and presence of fibrillation potential/positive sharp waves on EMG. The suspected clinical diagnosis may also have a significant impact. Clinicians should encourage MB testing, especially in cases with a strong clinical, laboratory, and electrodiagnostic suspicion and absence of genetic testing for the suspected myopathy.

\section{Funding}

None.

\section{Conflict of Interest}

None declared.

\section{References}

1 Gilchrist JM, Sachs GM. Electrodiagnostic studies in the management and prognosis of neuromuscular disorders. Muscle Nerve 2004;29(2):165-190

2 Buchthal F, Kamieniecka Z. The diagnostic yield of quantified electromyography and quantified muscle biopsy in neuromuscular disorders. Muscle Nerve 1982;5(4):265-280

3 Emery AE. The muscular dystrophies. Lancet 2002;359 (9307):687-695
4 Rabie M, Jossiphov J, Nevo Y. Electromyography (EMG) accuracy compared to muscle biopsy in childhood. J Child Neurol 2007;22(7):803-808

5 Chang J, Park YG, Choi YC, Choi JH, Moon JH. Correlation of electromyogram and muscle biopsy in myopathy of young age. Arch Phys Med Rehabil 2011;92(5):780-784

6 Jamshidi R, Harrison MR, Lee H, Nobuhara KK, Farmer DL. Indication for pediatric muscle biopsy determines usefulness. J Pediatr Surg 2008;43(12):2199-2201

7 Dubowitz V, Oldfors A, Sewry CA, The procedure of muscle biopsy. In: Dubowitz V, Sewry CA, Oldfors A eds. Muscle Biopsy: A Practical Approach. 4th ed. China: Saunders-Elsevier; $20132-15$

8 Miller S, Shevell M, Silver K, Kramer M; The Montreal Children's Hospital Neuromuscular Group. The diagnostic yield of the nerve-muscle skin biopsy in paediatric neurology practice. Pediatr Rehabil 1998;2(2):95-100

9 Gibreel WO, Selcen D, Zeidan MM, Ishitani MB, Moir CR, Zarroug AE. Safety and yield of muscle biopsy in pediatric patients in the modern era. J Pediatr Surg 2014;49(9):1429-1432

10 Werneck LC, Lima JG. Muscle biopsy correlated with electromyography. Study of 100 cases. Arq Neuropsiquiatr 1988;46(2):156-165

11 David WS, Jones HR, Jr. Electromyography and biopsy correlation with suggested protocol for evaluation of the floppy infant. Muscle Nerve 1994;17(4):424-430 